\title{
DYNAMIC ANALYSIS AND CONDITION ASSESSMENT OF RC BUILDING BY VARYING MATERIAL DAMPING AND TIME DEPENDENT PROPERTIES
}

\author{
Latha $\mathbf{H} \mathbf{R}^{1}$, Syed Ahamed ${ }^{2}$ \\ ${ }^{I}$ Post Graduate Student, Civil Engineering, Ghousia College of Engineering, Ramanagaram, Karnataka, India \\ ${ }^{2}$ Assistant Professor, Civil Engineering, Ghousia College of Engineering, Ramanagaram, Karnataka, India
}

\begin{abstract}
In the present dissertation study, an SMRF building situated in seismic zone-III having G+ 10 storey has been considered for performing linear dynamic or response spectrum method of analysis, to achieve and compare various results such as storey drift, storey displacement for considered DCON-2 and DCON-3 load combinations as per IS: 1893-2002 (Part-I), all imposed load are considered as per IS 875:2000. Also the condition assessment of the building models through system identification approach and extract the modal identification parameters like frequency, damping, mode shapes. Furthermore spectral displacement, spectral velocity and spectral acceleration are obtained for various percentages of damping i.e., 0, 5 and 10, for all the considered SMRF building models. The overall dissertation flow has been carried out to perform linear dynamic analysis (response spectrum method) for the parameters ELCENTRO, using the finite element method based analytical software ETABS-15 version.
\end{abstract}

Keywords: Linear dynamic, Condition assessment, Material damping, Time dependent and Response spectrum

\section{INTRODUCTION}

\subsection{General}

Earthquake is the faction or trembling caused due to the sudden discharge of energy in the earth's crust, the sudden discharge of energy is due to the movement of rocks in the earth's crust. Depending on magnitude, intensity and effect of the earthquake may small or large earthquake. Large earthquake usually begin with slight tremors but rapidly take from of one or more violent shocks. The subterranean point of source of an earthquake is called its focus; the point on the plane directly over the focus is the epicenter. In some parts of the world, earthquake is related with volcanic activity. As most of the earthquake occur from stress buildup due to deformation of the earth's crust, and also calls upon the knowledge of the physics of the earth. There are three main types of geological fault that may cause an earthquake: normal, reverse and strike-slip. Normal faults occur in areas where the crust is being extended. Reverse faults occur in areas where the crust is being shortened. Strike-slip faults are steep structures where the two sides of the faults slip horizontally past each other.

Condition assessment is very important as a measure of safety, it is nothing but assessing the condition of the structures frequently. By doing the dynamic analysis we get the frequency response functions which are used in assessing and analyzing the damage in the structure, its load-bearing capacity and serviceability are assessed, and any damage to structural elements is detected.

\subsection{Objectives}

- To carry out linear static (Equivalent static method) and linear dynamic (response spectrum method) analysis for the considered SMRF building models situated in zoneIII as per IS 1893:2002 Part-I for the considered load combinations.

- To evaluate the condition of the considered SMRF building model using system identification approach.

- To execute linear dynamic analysis to obtain the modal identification parameters such as frequency, damping and mode shapes.

- To study the varying effect of material damping and time dependent properties for the considered SMRF building models.

- To perform linear dynamic response spectrum method of analysis and obtain various results like storey drift, storey displacements are tabulated and are plotted on graph.

- Linear dynamic parameters such as spectral displacement, spectral velocity and spectral acceleration are obtained for various percentages of damping i.e., 0 , 5 and 10, for all the considered SMRF building models.

\subsection{Methods of Analysis}

1. Linear static analysis

2. Linear dynamic analysis (Response spectrum method)

The above two method of analysis is carried out using ETABS-2015 software. Linear static method is adopted to obtain the retort of a building. In this method the total design lateral force along any principal direction are obtained. In response spectrum method of analysis the 
response of the structure to the several earthquake motions is obtained in the form of smooth design spectra. This method gives the maximum displacements, design seismic force, base shear storey drift for each mode of vibration.

\section{MODELLING AND ANALYSIS}

Table-1: Model Details

\begin{tabular}{|l|l|}
\hline Structure Type & SMRF \\
\hline No of storeys & 11 \\
\hline Height of the building & 33.5 \\
\hline Materials & $\begin{array}{l}\text { Concrete M30 and Steel } \\
\text { Fe500 }\end{array}$ \\
\hline Plan Dimension & $36 \mathrm{~m} \times 20 \mathrm{~m}$ \\
\hline No of bay in X direction & 6 \\
\hline No of bay in Y direction & 4 \\
\hline Size of Column & $500 \times 600 \mathrm{~mm}$ \\
\hline Size of Beam & $400 \times 600 \mathrm{~mm}$ \\
\hline Thickness of Slab & $150 \mathrm{~mm}$ \\
\hline Density of Concrete & $25 \mathrm{Kn} / \mathrm{m}^{2}$ \\
\hline Poisson's Ratio & 0.25 \\
\hline Seismic Zone & III \\
\hline Zone factor(Z) & 0.16 \\
\hline Soil Type & II(Medium soil) \\
\hline Importance Factor(I) & 1.5 \\
\hline $\begin{array}{l}\text { Response } \\
\text { Factor(R) }\end{array}$ & 5 \\
\hline Model-1 & Bare frame type \\
\hline Model-2 & Material damping type \\
\hline Model-3 & Time dependent type \\
\hline
\end{tabular}

Steps involved to carry out this analysis is as follows

- Modeling of the finite elemental frame.

- Define and assign the properties of beams, column and slabs.

- Define and assign the static load combination.

- Carryout linear static analysis.

- Dynamic analysis is carryout by using Response spectrum method.

- Displacement, time period, frequency response are obtained which are verified and validated



Fig 1: Plan of the frame model from ETABS-2015



Fig 2: 3D view of the frame model from ETABS-2015

\section{LINEAR DYNAMIC RESULTS}

\subsection{Storey Displacement}

Table- 2: Storey displacement of all models in X direction

\begin{tabular}{|l|l|l|l|}
\hline \multirow{2}{*}{$\begin{array}{l}\text { Storey } \\
\text { no }\end{array}$} & Model-1 & Model-2 & Model-3 \\
\cline { 2 - 4 } & RSX & RSX & RSX \\
\hline 1 & 16.5 & 15 & 16 \\
\hline 2 & 16.1 & 14.7 & 15.7 \\
\hline 3 & 15.5 & 14.2 & 15.1 \\
\hline 4 & 14.5 & 13.4 & 14.2 \\
\hline 5 & 13.3 & 12.3 & 13 \\
\hline 6 & 11.9 & 11 & 11.6 \\
\hline 7 & 10.3 & 9.6 & 10 \\
\hline 8 & 8.4 & 7.9 & 8.3 \\
\hline 9 & 6.4 & 6 & 6.3 \\
\hline 10 & 4.2 & 4 & 4.1 \\
\hline 11 & 1.9 & 1.9 & 1.9 \\
\hline
\end{tabular}



\section{Storey no}

Chart 1: Storey displacement curve of all models in $X$ direction 


\subsection{Storey Drift}

Table-3: Storey drifts of all models in $\mathrm{X}$ direction

\begin{tabular}{|l|l|l|l|}
\hline \multirow{2}{*}{$\begin{array}{l}\text { Storey } \\
\text { no }\end{array}$} & Model-1 & Model-2 & Model-3 \\
\cline { 2 - 4 } & RSX & RSX & RSX \\
\hline 1 & 0.000148 & 0.000122 & 0.00014 \\
\hline 2 & 0.000271 & 0.000226 & 0.000257 \\
\hline 3 & 0.000383 & 0.000328 & 0.000366 \\
\hline 4 & 0.00047 & 0.000412 & 0.000453 \\
\hline 5 & 0.000538 & 0.00048 & 0.000521 \\
\hline 6 & 0.000594 & 0.000537 & 0.000577 \\
\hline 7 & 0.000645 & 0.000589 & 0.000628 \\
\hline 8 & 0.000694 & 0.000641 & 0.000677 \\
\hline 9 & 0.000738 & 0.000689 & 0.000722 \\
\hline 10 & 0.000754 & 0.000713 & 0.000739 \\
\hline 11 & 0.000556 & 0.000533 & 0.000547 \\
\hline
\end{tabular}

\section{Storey Drift}

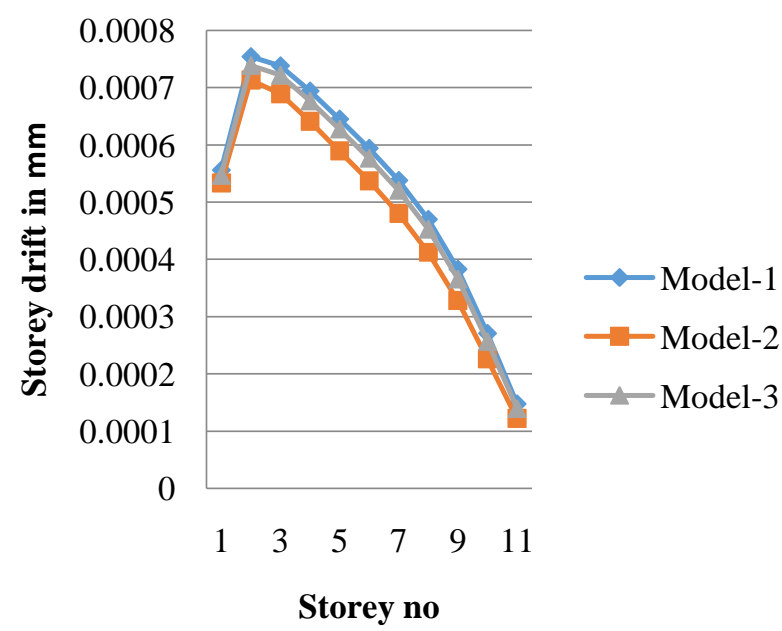

Chart 2: Storey drift curve of all models in X direction

\subsection{Base Spectral Displacement}

Table-4: Base Spectral displacement for model-1

\begin{tabular}{|l|l|l|l|}
\hline \multirow{2}{*}{$\begin{array}{l}\text { Period } \\
(\mathbf{s e c})\end{array}$} & \multicolumn{3}{|l}{$\begin{array}{l}\text { Spectral Displacement for model-1 } \\
\text { in mm }\end{array}$} \\
\cline { 2 - 4 } & $\begin{array}{l}\text { Damping } \\
\text { 0 }\end{array}$ & $\begin{array}{l}\text { Damping } \\
\mathbf{0 . 0 5}\end{array}$ & $\begin{array}{l}\text { Damping } \\
\mathbf{0 . 1}\end{array}$ \\
\hline 0.03 & 0.2 & 0.2 & 0.2 \\
\hline 0.04 & 0.4 & 0.4 & 0.4 \\
\hline 0.05 & 0.6 & 0.7 & 0.7 \\
\hline 0.1 & 2.6 & 2.7 & 2.6 \\
\hline 0.15 & 10.8 & 9.4 & 8.1 \\
\hline 0.2 & 24.5 & 7.2 & 7.1 \\
\hline 0.25 & 31.6 & 26.2 & 20.8 \\
\hline 0.5 & 64.8 & 63.5 & 59.8 \\
\hline 1 & 214.5 & 202.7 & 187.8 \\
\hline 2 & 430.2 & 432.5 & 432.1 \\
\hline 5 & 2725.6 & 2631.4 & 2555.5 \\
\hline
\end{tabular}

\section{Spectral Displacement}

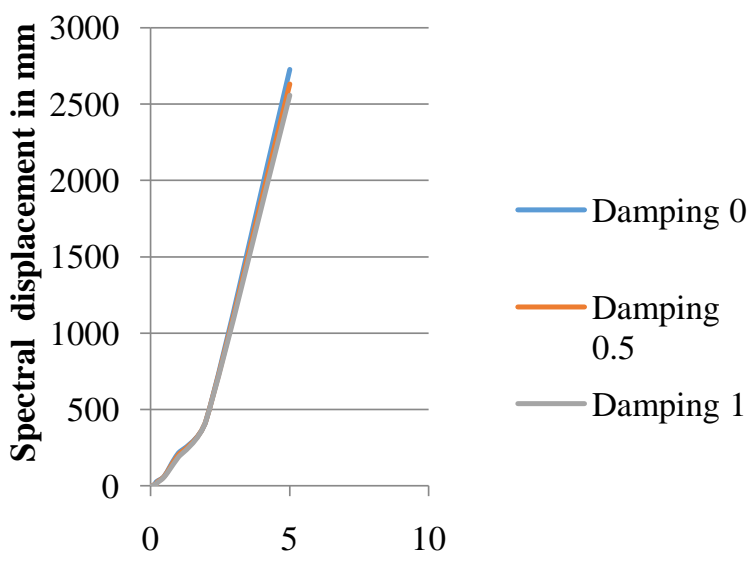

Period, sec

Chart 3: Base spectral displacement curve for model-1

Table-5: Base Spectral displacement for model-1

\begin{tabular}{|l|l|l|l|}
\hline \multirow{2}{*}{$\begin{array}{l}\text { Period } \\
(\mathbf{s e c})\end{array}$} & \multicolumn{3}{|l}{$\begin{array}{l}\text { Spectral Displacement for model- } \\
\text { 2 in mm }\end{array}$} \\
\cline { 2 - 4 } & $\begin{array}{l}\text { Damping } \\
\text { 0 }\end{array}$ & $\begin{array}{l}\text { Damping } \\
\mathbf{0 . 0 5}\end{array}$ & $\begin{array}{l}\text { Dampin } \\
\text { g 0.1 }\end{array}$ \\
\hline 0.03 & 0.5 & 0.5 & 0.5 \\
\hline 0.04 & 0.8 & 0.9 & 0.9 \\
\hline 0.05 & 1.3 & 1.4 & 1.4 \\
\hline 0.1 & 5.3 & 5.4 & 5.4 \\
\hline 0.15 & 22.1 & 19.5 & 16.7 \\
\hline 0.2 & 67.7 & 13 & 14.1 \\
\hline 0.25 & 64.8 & 53.8 & 42.7 \\
\hline 0.5 & 133.1 & 130.4 & 122.9 \\
\hline 1 & 440.3 & 416.2 & 385.6 \\
\hline 2 & 883.3 & 888 & 887.2 \\
\hline 5 & 5596.1 & 5402.6 & 5246.7 \\
\hline & & & \\
\hline
\end{tabular}

\section{Spectral Displacement}

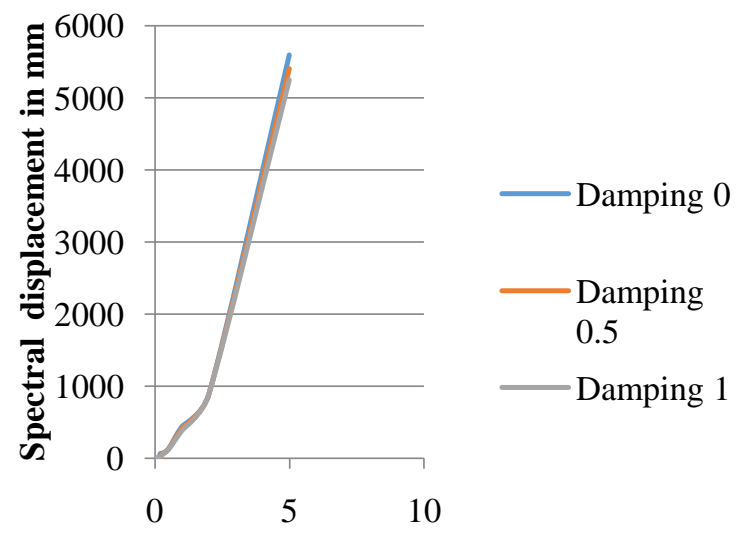

Period, sec

Chart 4: Base spectral displacement curve for model-2 
Table-6: Base Spectral displacement for model-3

\begin{tabular}{|c|c|c|c|}
\hline \multirow{2}{*}{$\begin{array}{l}\text { Perio } \\
\text { d } \\
(\mathrm{sec})\end{array}$} & \multicolumn{3}{|c|}{$\begin{array}{l}\text { Spectral Displacement for } \\
\text { model-3 in mm }\end{array}$} \\
\hline & $\begin{array}{l}\text { Dampin } \\
\text { g o }\end{array}$ & $\begin{array}{l}\text { Damping } \\
0.05\end{array}$ & $\begin{array}{l}\text { Damping } \\
\text { 0.1 }\end{array}$ \\
\hline 0.03 & 0.4 & 0.4 & 0.4 \\
\hline 0.04 & 0.7 & 0.7 & 0.7 \\
\hline 0.05 & 1.1 & 1.1 & 1.1 \\
\hline 0.1 & 4.5 & 4.6 & 4.5 \\
\hline 0.15 & 18.5 & 16.2 & 14 \\
\hline 0.2 & 42.2 & 12.3 & 12.2 \\
\hline 0.25 & 54.3 & 45.1 & 35.8 \\
\hline 0.5 & 111.5 & 109.3 & 103 \\
\hline 1 & 369 & 348.8 & 323.2 \\
\hline 2 & 740.3 & 744.2 & 743.5 \\
\hline 5 & 4690 & 4527.8 & 4397.2 \\
\hline
\end{tabular}



Period, sec

Chart 5: Base spectral displacement curve for model-3

\subsection{Spectral Velocity}

Table-7: Base Spectral velocity for model-1

\begin{tabular}{|l|l|l|l|}
\hline \multirow{2}{*}{$\begin{array}{l}\text { Period } \\
\text { (sec) }\end{array}$} & \multicolumn{2}{l}{$\begin{array}{l}\text { Spectral velocity for model-1 in } \\
\text { mm/sec }\end{array}$} \\
\cline { 2 - 4 } & $\begin{array}{l}\text { Damping } \\
\text { 0 }\end{array}$ & $\begin{array}{l}\text { Damping } \\
\text { 0.05 }\end{array}$ & $\begin{array}{l}\text { Damping } \\
\text { 0.1 }\end{array}$ \\
\hline 0.03 & 4.56 & 2.52 & 2.43 \\
\hline 0.04 & 346.14 & 11.06 & 6.42 \\
\hline 0.05 & 0 & 2.7 & 4.5 \\
\hline 0.1 & 0 & 5.54 & 10.85 \\
\hline 0.15 & 95.72 & 113.49 & 100.56 \\
\hline 0.2 & 8653.47 & 1059.68 & 590.64 \\
\hline 0.25 & 484.33 & 430.22 & 312.5 \\
\hline 0.5 & 447.66 & 428.94 & 422.39 \\
\hline 1 & 889.12 & 905.63 & 900.97 \\
\hline 2 & 485.8 & 437.16 & 428.89 \\
\hline 5 & 887.42 & 845.59 & 801.76 \\
\hline
\end{tabular}

\section{Spectral velocity}

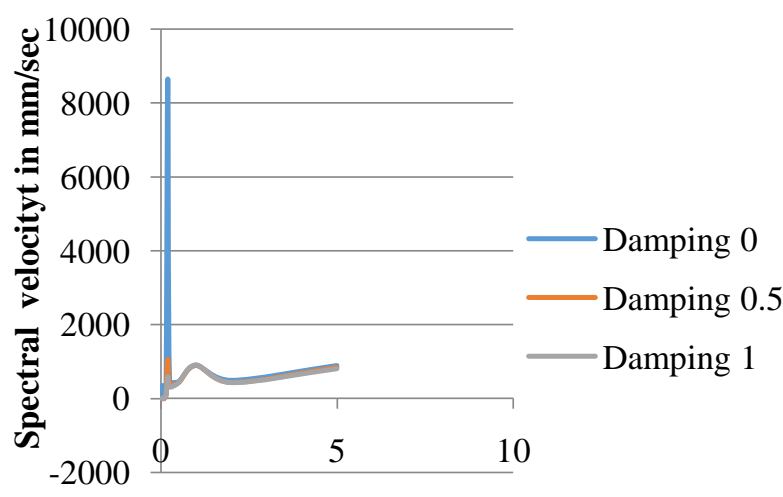

Period, sec

Chart 6: Base spectral velocity curve for model-1

Table-8: Base Spectral velocity for model-2

\begin{tabular}{|l|l|l|l|}
\hline \multirow{2}{*}{$\begin{array}{l}\text { Perio } \\
\text { d } \\
(\mathbf{s e c})\end{array}$} & \multicolumn{3}{l}{$\begin{array}{l}\text { Spectral velocity for model-2 in } \\
\text { mm/sec }\end{array}$} \\
\cline { 2 - 4 } & $\begin{array}{l}\text { Damping } \\
\text { 0 }\end{array}$ & $\begin{array}{l}\text { Damping } \\
\mathbf{0 . 0 5}\end{array}$ & $\begin{array}{l}\text { Damping } \\
\mathbf{0 . 1}\end{array}$ \\
\hline 0.03 & 9.36 & 5.17 & 5 \\
\hline 0.04 & 710.67 & 22.71 & 13.18 \\
\hline 0.05 & 0 & 5.55 & 9.25 \\
\hline 0.1 & 0 & 11.37 & 22.29 \\
\hline 0.15 & 194.06 & 236.17 & 208.04 \\
\hline 0.2 & 17601.67 & 2177.78 & 1214.22 \\
\hline 0.25 & 994.39 & 883.3 & 641.6 \\
\hline 0.5 & 919.1 & 880.68 & 867.21 \\
\hline 1 & 1825.48 & 1859.38 & 1849.81 \\
\hline 2 & 997.4 & 897.55 & 880.57 \\
\hline 5 & 1821.98 & 1736.11 & 1646.12 \\
\hline
\end{tabular}

\section{Spectral velocity}

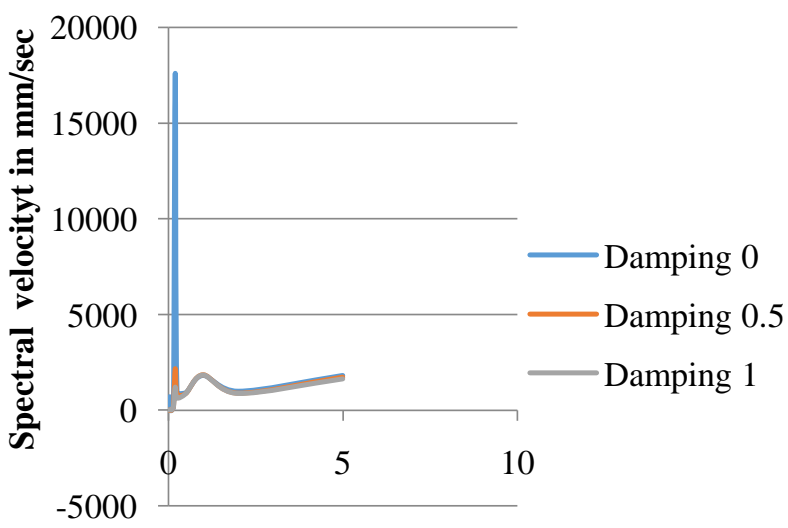

Period, sec

Chart 7: Base spectral velocity curve for model-2 
Table-9: Base Spectral velocity for model-3

\begin{tabular}{|c|c|c|c|}
\hline \multirow{2}{*}{$\begin{array}{l}\text { Period } \\
\text { (sec) }\end{array}$} & \multicolumn{3}{|c|}{$\begin{array}{l}\text { Spectral velocity for model-3 in } \\
\mathrm{mm} / \mathrm{sec}\end{array}$} \\
\hline & $\begin{array}{l}\text { Damping } \\
\text { 0 }\end{array}$ & $\begin{array}{l}\text { Damping } \\
0.05\end{array}$ & $\begin{array}{l}\text { Damping } \\
0.1\end{array}$ \\
\hline 0.03 & 7.84 & 4.33 & 4.19 \\
\hline 0.04 & 595.6 & 19.03 & 11.05 \\
\hline 0.05 & 0 & 4.65 & 7.75 \\
\hline 0.1 & 0 & 9.53 & 18.68 \\
\hline 0.15 & 164.71 & 195.29 & 173.03 \\
\hline 0.2 & 14889.97 & 1823.38 & 1016.31 \\
\hline 0.25 & 833.38 & 740.28 & 537.72 \\
\hline 0.5 & 770.29 & 738.08 & 726.8 \\
\hline 1 & 1529.9 & 1558.32 & 1550.3 \\
\hline 2 & 835.91 & 752.23 & 737.99 \\
\hline 5 & 1526.97 & 1455 & 1379.58 \\
\hline
\end{tabular}

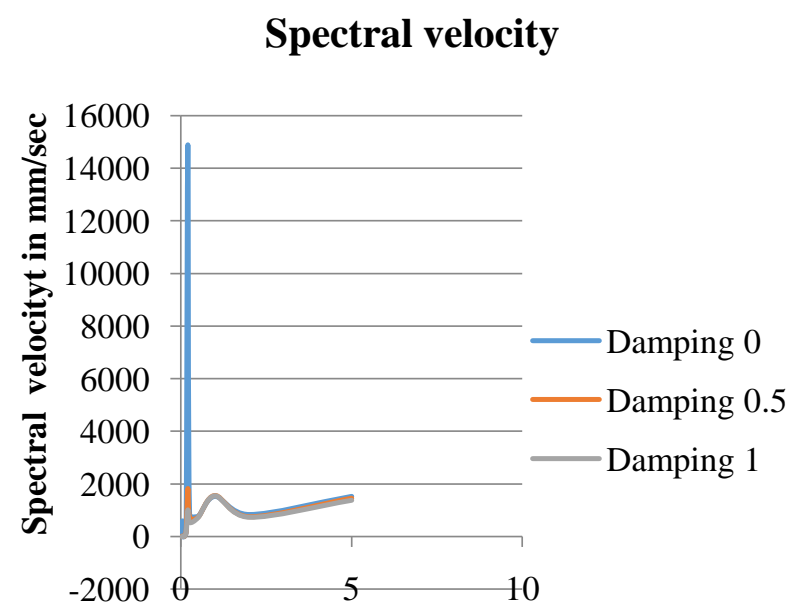

Period, sec

Chart 8: Base spectral velocity curve for model-3

\subsection{Spectral Acceleration}

Table-10: Base Spectral acceleration for model-1

\begin{tabular}{|l|l|l|l|}
\hline \multirow{2}{*}{$\begin{array}{l}\text { Period } \\
(\text { sec })\end{array}$} & \multicolumn{3}{|l}{$\begin{array}{l}\text { Spectral acceleration for model-1 } \\
\text { in mm/sec }\end{array}$} \\
\cline { 2 - 4 } & $\begin{array}{l}\text { Damping } \\
\text { 0 }\end{array}$ & $\begin{array}{l}\text { Damping } \\
\text { 0.05 }\end{array}$ & $\begin{array}{l}\text { Damping } \\
\text { 0.1 }\end{array}$ \\
\hline 0.03 & 10037.15 & 10131.04 & 10369.24 \\
\hline 0.04 & 10212.21 & 10461.24 & 10463.48 \\
\hline 0.05 & 10212.21 & 10503.31 & 10515.62 \\
\hline 0.1 & 10212.21 & 10504.05 & 10520.32 \\
\hline 0.15 & 18851.37 & 16811.89 & 14896.48 \\
\hline 0.2 & 10212.21 & 9794.56 & 9718.97 \\
\hline 0.25 & 19946.09 & 16013.17 & 12276.17 \\
\hline 0.5 & 10234.68 & 9967.52 & 9372.46 \\
\hline 1 & 8467.21 & 7854.08 & 7844.29 \\
\hline 2 & 4246.32 & 4261.77 & 4333.9 \\
\hline 5 & 4304.17 & 4231.2 & 4189.42 \\
\hline
\end{tabular}

\section{Spectral acceleration}

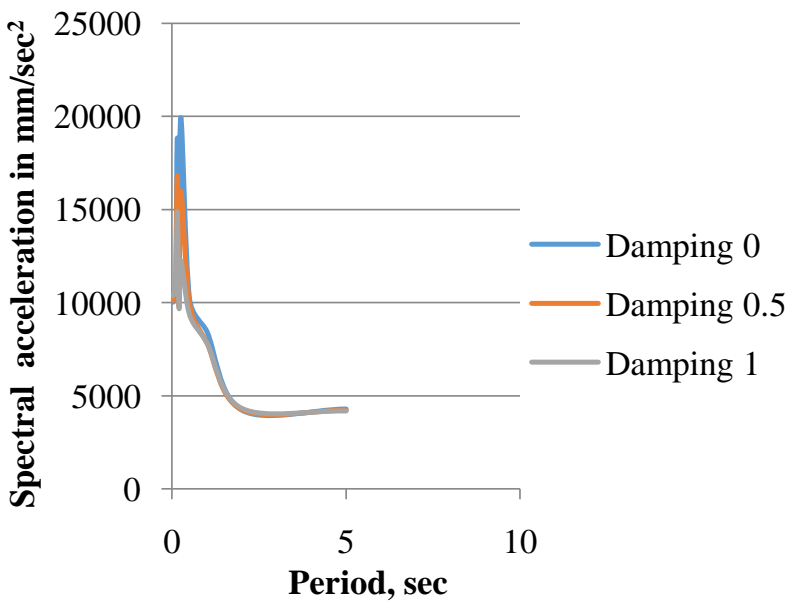

Chart 9: Base spectral acceleration curve for model-1

Table-11: Base Spectral acceleration for model-2

\begin{tabular}{|l|l|l|l|}
\hline \multirow{2}{*}{$\begin{array}{l}\text { Period } \\
\text { (sec) }\end{array}$} & \multicolumn{2}{|l}{$\begin{array}{l}\text { Spectral acceleration for model-2 } \\
\text { in mm/sec }\end{array}$} \\
\cline { 2 - 4 } & $\begin{array}{l}\text { Damping } \\
\text { 0 }\end{array}$ & $\begin{array}{l}\text { Damping } \\
\text { 0.05 }\end{array}$ & $\begin{array}{l}\text { Damping } \\
\text { 0.1 }\end{array}$ \\
\hline 0.03 & 20607.58 & 20800.34 & 21289.39 \\
\hline 0.04 & 20966.99 & 21478.28 & 21482.87 \\
\hline 0.05 & 20966.99 & 21564.66 & 21589.94 \\
\hline 0.1 & 20966.99 & 21566.17 & 21599.59 \\
\hline 0.15 & 38665.74 & 34595.98 & 30610.99 \\
\hline 0.2 & 66675.27 & 18807.47 & 19593.11 \\
\hline 0.25 & 40951.9 & 32877.1 & 25204.57 \\
\hline 0.5 & 21013.12 & 20464.62 & 19242.88 \\
\hline 1 & 17384.28 & 16125.44 & 16105.35 \\
\hline 2 & 8718.24 & 8749.96 & 8898.05 \\
\hline 5 & 8837.02 & 8687.19 & 8601.42 \\
\hline
\end{tabular}



Chart 10: Base spectral acceleration curve for model-2 
Table-12: Base Spectral acceleration for model-3

\begin{tabular}{|l|l|l|l|}
\hline \multirow{2}{*}{$\begin{array}{l}\text { Perio } \\
\text { d } \\
(\mathbf{s e c})\end{array}$} & \multicolumn{4}{|l}{$\begin{array}{l}\text { Spectral acceleration for model- } \\
\text { 3 in mm/sec }\end{array}$} \\
\cline { 2 - 4 } & $\begin{array}{l}\text { Dampin } \\
\text { g 0 }\end{array}$ & $\begin{array}{l}\text { Dampin } \\
\text { g 0.05 }\end{array}$ & $\begin{array}{l}\text { Dampin } \\
\text { g 0.1 }\end{array}$ \\
\hline 0.03 & 17270.86 & 17432.41 & 17842.28 \\
\hline 0.04 & 17572.08 & 18000.58 & 18004.43 \\
\hline 0.05 & 17572.08 & 18072.97 & 18094.16 \\
\hline 0.1 & 17572.08 & 18074.25 & 18102.25 \\
\hline 0.15 & 32437.43 & 28928.11 & 25632.28 \\
\hline 0.2 & 17572.08 & 16853.43 & 16723.37 \\
\hline 0.25 & 34321.09 & 27553.75 & 21123.53 \\
\hline 0.5 & 17610.74 & 17151.05 & 16127.14 \\
\hline 1 & 14569.47 & 13514.46 & 13497.62 \\
\hline 2 & 7306.61 & 7333.19 & 7457.31 \\
\hline 5 & 7406.16 & 7280.59 & 7208.71 \\
\hline
\end{tabular}

Spectral acceleration

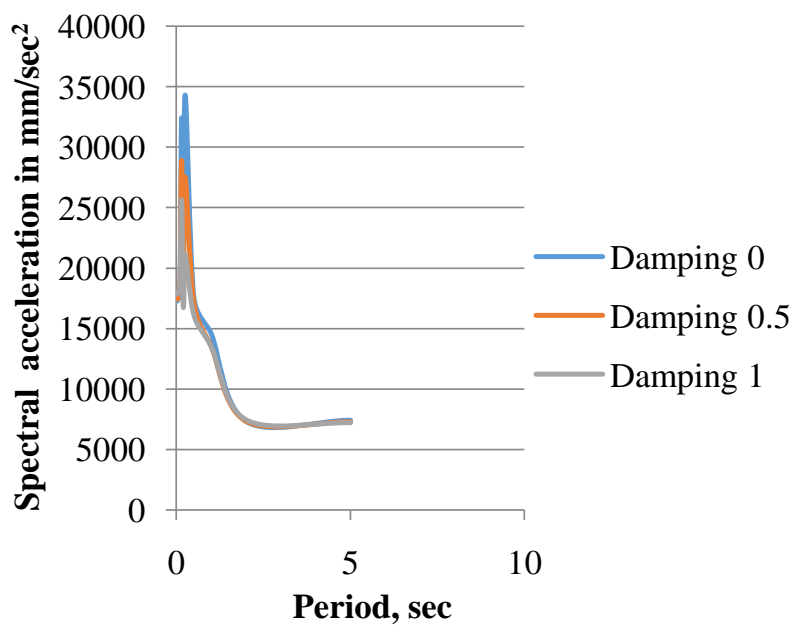

Chart 11: Storey acceleration curve for model-3

Tripartite plot

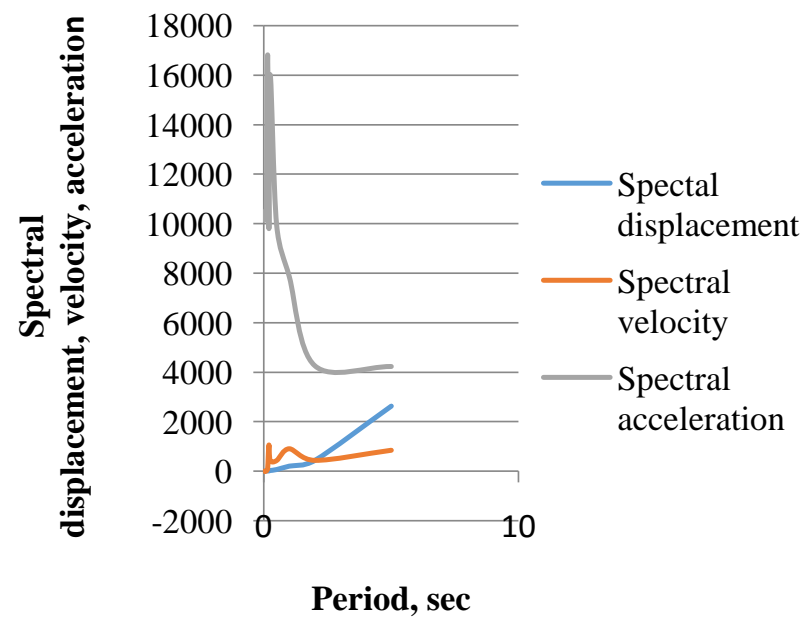

Chart 12: Tripartite plot for model-1
Tripartite plot

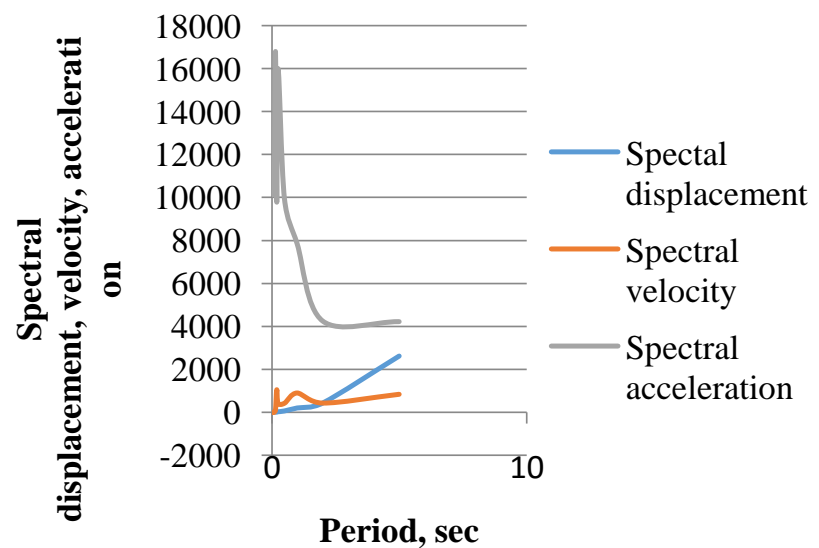

Chart 13: Tripartite plot for model-2

Tripartite plot

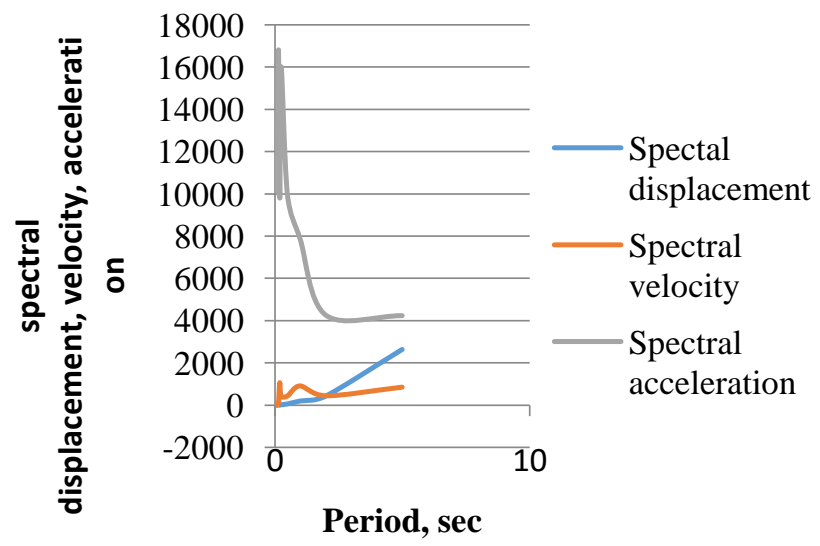

Chart 14: Tripartite for model-3

\subsection{Time Period and Frequency}

Table 13: Time period and Frequency of all models

\begin{tabular}{|l|l|l|}
\hline $\begin{array}{l}\text { Storey } \\
\text { no }\end{array}$ & $\begin{array}{l}\text { Time period } \\
\text { (sec) }\end{array}$ & $\begin{array}{l}\text { Frequency } \\
\text { (cycle/sec) }\end{array}$ \\
\hline 1 & 1.689 & 0.592 \\
\hline 2 & 1.605 & 0.623 \\
\hline 3 & 1.512 & 0.661 \\
\hline 4 & 0.554 & 1.804 \\
\hline 5 & 0.524 & 1.907 \\
\hline 6 & 0.497 & 2.013 \\
\hline 7 & 0.32 & 3.122 \\
\hline 8 & 0.302 & 3.311 \\
\hline 9 & 0.289 & 3.46 \\
\hline 10 & 0.222 & 4.503 \\
\hline 11 & 0.206 & 4.843 \\
\hline 12 & 0.2 & 5.002 \\
\hline 13 & 0.167 & 5.981 \\
\hline 14 & 0.153 & 6.529 \\
\hline 15 & 0.15 & 6.662 \\
\hline 16 & 0.133 & 7.528 \\
\hline 17 & 0.12 & 8.348 \\
\hline 18 & 0.119 & 8.415 \\
\hline 19 & 0.11 & 9.108 \\
\hline & & \\
\hline
\end{tabular}




\begin{tabular}{|l|l|l|}
\hline 20 & 0.098 & 10.22 \\
\hline 21 & 0.098 & 10.254 \\
\hline 22 & 0.094 & 10.648 \\
\hline 23 & 0.083 & 11.996 \\
\hline 24 & 0.083 & 12.055 \\
\hline 25 & 0.082 & 12.163 \\
\hline 26 & 0.076 & 13.206 \\
\hline 27 & 0.073 & 13.633 \\
\hline 28 & 0.072 & 13.95 \\
\hline 29 & 0.072 & 13.971 \\
\hline 30 & 0.067 & 14.983 \\
\hline 31 & 0.065 & 15.443 \\
\hline 32 & 0.063 & 15.884 \\
\hline 33 & 0.061 & 16.449 \\
\hline
\end{tabular}

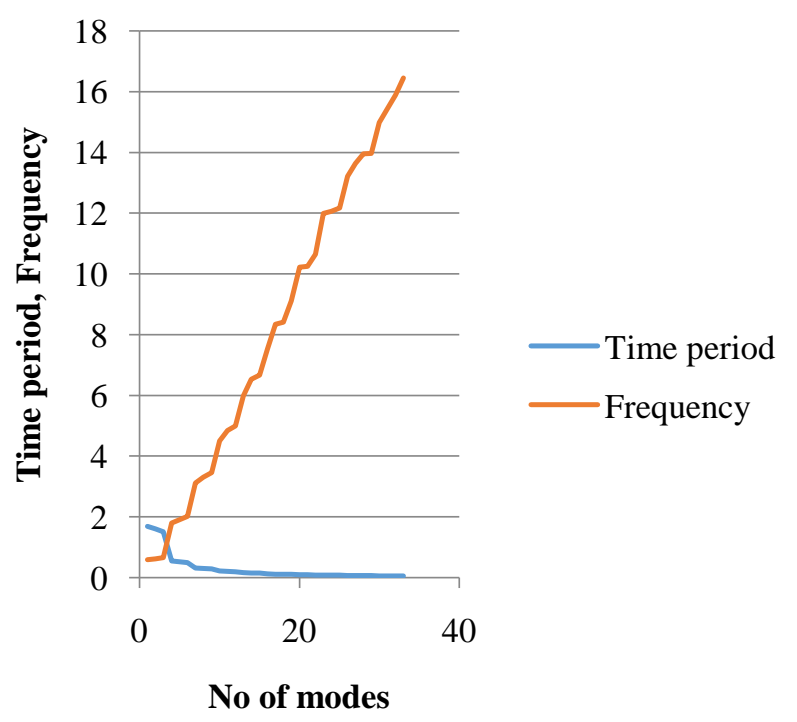

Chart 1: Time period and frequency curve for all models

\subsection{Mode Shapes}



Fig 3: Mode shape for $1^{\text {st }}$ mode from ETABS-2015

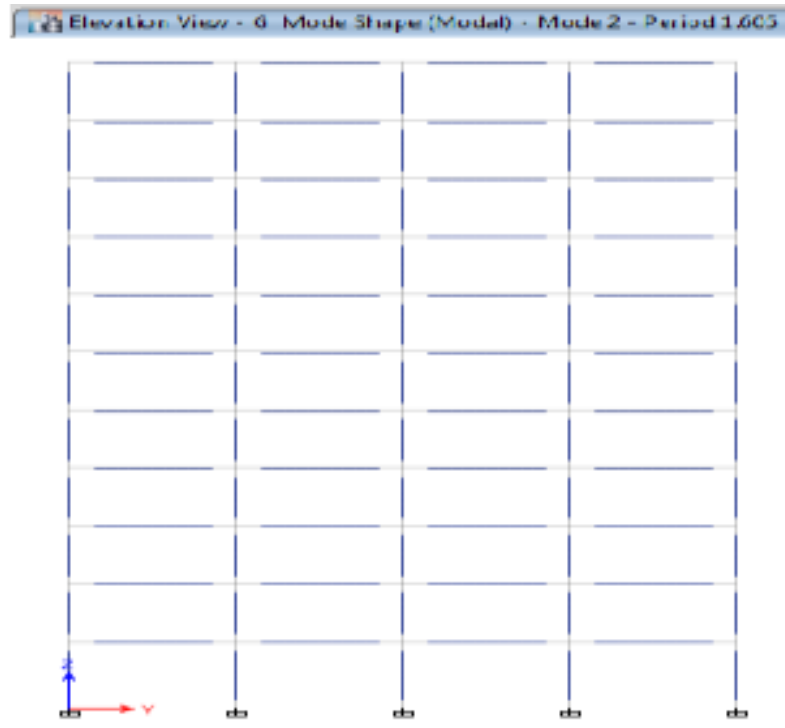

Fig 4: Mode shape for $2^{\text {nd }}$ mode from ETABS-2015



Fig 5: Mode shape for $3^{\text {rd }}$ mode from ETABS-2015



Fig 6: Mode shape for $4^{\text {th }}$ mode from ETABS-2015 


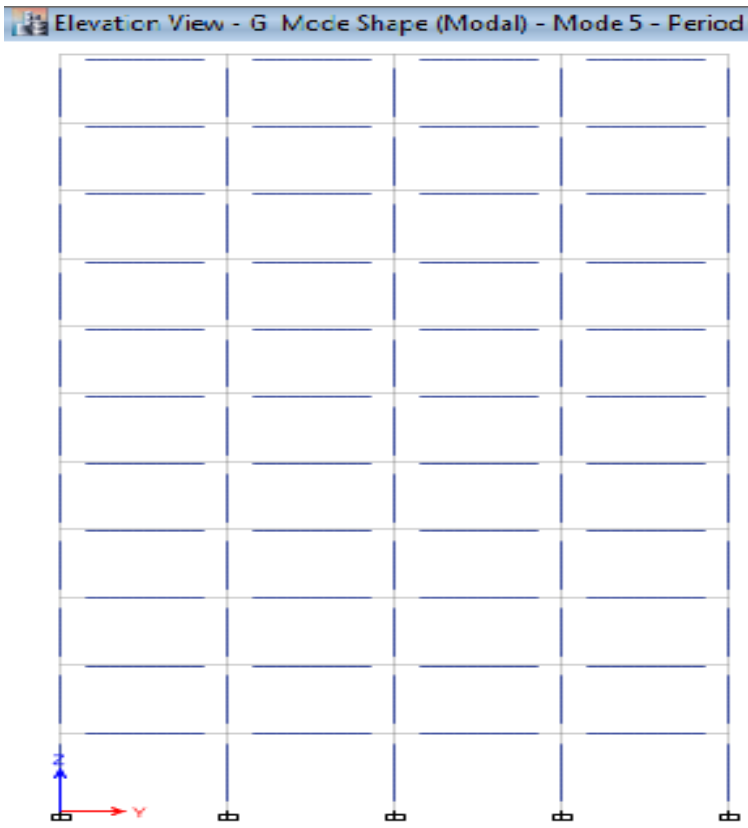

Fig 7: Mode shape for $5^{\text {th }}$ mode from ETABS-2015



Fig 8: Mode shape for $6^{\text {th }}$ mode from ETABS-2015

\section{CONCLUSION}

- $\quad$ The storey displacement, storey drift for DCON-3 load combination is greater than storey displacement, storey drift of DCON-2 load combination for equivalent linear static analysis, obtained for the models under consideration.

- The linear dynamic response spectrum method of analysis is performed and results yields that the model (model-2) with material damping properties is displaced less rather than the time dependent model (model-3) and bare frame model (model-1).

- The various response spectrum results like spectral displacement, spectral velocity and spectral acceleration are obtained for various percentage of damping with respect to period.
- The results like time period, frequency and mode shapes are same for all the three models are tabulated and graph are plotted.

- The results such as frequency is inversely proportional to time period, i.e., as the time period goes on decreases the corresponding frequency goes on increases.

- It is seen that the model-2 with the material damping properties yields better response and results in comparison with all other models.

\section{REFERENCES}

[1] Jerry E. Stephens and James T.P. Yao (1987) "Damage Assessment Using Response measurement".

[2] D.N.Farhey and A.E.Aktan (1996) "Condition Assessment for Seismic Evaluation".

[3] Hjelmstad and S.Shin (1997) "Damage Detection and Assessment of Structures from Static Response".

[4] Federal Emergency Management Agency (FEMA 273) NEHRP GUIDLINES (1997),

[5] Federal emergency management agency (FEMA 356) Nov 2000 "Pre Standard and Commentary for the Seismic Rehabilitation of Building".

[6] Koen Van Den Abeelea, Joelle De Visscher (2000) paper entitled "Damage Assessment in Reinforced Concrete Using Spectral and Temporal Non-Linear Vibration Techniques".

[7] IS 1893-2002 (Part-1) "Criteria for Earthquake Resistant Design of Structures".

[8] IS 875-2000, "Code of Practice for Design Loads (other than earthquake)"

\section{BIOGRAPHIES}

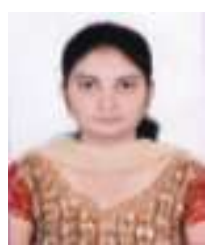

Latha H R, Post graduate student, Ghousia college of engineering, Ramanagaram562159, Karnataka, India.

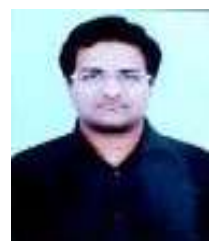

Syed Ahamed, Assistant Professor, Ghousia college of engineering, Ramanagram-562159, Karnataka, India. 